\title{
Zika virus, a novel mosquito-borne congenital virus infection
}

\author{
J. David Beckham ${ }^{1}$
}

Received: 30 March 2017 / Accepted: 30 March 2017 /Published online: 20 April 2017

(C) Journal of NeuroVirology, Inc. 2017

Throughout human history of infectious diseases, we have encountered a myriad of novel infectious diseases that have challenged our scientific and health-care responses. Early success with vaccine preventable diseases like small pox, measles, and polio virus support our ability to prevent and sometimes eradicate human-dependent transmission of important viral infectious diseases. Subsequent emergence of nonvaccine preventable virus infections that depend on human sexual transmission such as human immunodeficiency virus (HIV) have challenged our ability to develop a response to control an epidemic using therapeutic approaches instead of vaccines. The advent and development of antiretroviral therapies (ART) for HIV have dramatically changed our approach to treating viral infections. Currently, we treat HIV-infected patients as a chronic disease for the lifetime of a patient. While the outcomes for HIV have dramatically improved with ART, a cure or vaccine for HIV remains important priorities.

Similarly, the emergence and spread of some arthropodborne virus (arbovirus) infections like dengue virus and West Nile virus (WNV) have proven to be challenging to control using vaccine approaches for very different reasons. In the case of dengue virus, the existence of four serotypes and cross-reactive antibodies that can result in antibody-dependent enhancement (ADE) of infection with heterologous dengue isolates makes vaccine safety more difficult. Following initial infection with any of the four serotypes of dengue, symptoms are usually characterized as a febrile illness associated with myalgias (body aches), arthralgias (joint aches), and

J. David Beckham

david.beckham@ucdenver.edu

1 Departments of Medicine/Infectious Diseases, Neurology, and Immunologyand Microbiology, University of Colorado School of Medicine, Aurora, CO, USA sometimes a rash. In general, most patients recover without sequelae following initial exposure to dengue virus. As discussed by Singh et al., ADE occurs when an individual is infected with a serotype of dengue virus, for example dengue 2 , and develops neutralizing antibodies to that specific serotype (Singh et al. 2017). The antibodies specific for dengue 2 can cross-react with other serotypes of dengue (1, 3, and 4) following infection, and the circulating dengue 2 antibodies can bind immature, non-infectious heterologous dengue virions. These virions are not otherwise infectious, but with a heterologous dengue 2 antibody attached, these immature virions are now endocytosed by Fc-receptors on myeloid cells resulting in amplification of infectious particles. This mechanism is thought to significantly contribute to the severe clinical consequences of repeat dengue infection from heterologous serotypes resulting in dengue shock syndrome and other complications. So, the existence of four dengue serotypes and the subsequent potential for ADE has hampered development of safe and efficacious dengue virus vaccines.

Development of a vaccine to WNV has not been technically challenging like dengue virus. Several vaccines currently exist and are safe and immunogenic (Brandler and Tangy 2013). However, clinical trials and targeted vaccine approaches have been significantly hampered by the sporadic epidemiology of WNV outbreaks. Since outbreaks of WNV disease and associated meningitis, encephalitis, and myelitis occur as seasonal epidemics, the location and potential case numbers have been difficult to predict. Because of this sporadic epidemiology, designing clinical trials to test efficacy of potential WNV vaccines or therapeutic approaches has been difficult because enrollment numbers are too small to support a conclusion based on sufficiently statistically powered data.

Recently, Zika virus (ZIKV) has emerged and spread across the globe. As discussed by Zhou et al. and Singh et al. in this issue of the Journal of Neurovirology, the emergence of ZIKV 
presents challenges for scientific and health-care communities that share unique features from the viruses mentioned above (Singh et al. 2017; Zhou et al. 2017). Like WNV, ZIKV is neuroinvasive but is the first known arbovirus that is clearly associated with neuroinvasion of the fetal central nervous system (CNS) resulting in severe congenital anomalies (Honein et al. 2017). This is a novel phenotype in the field of arbovirus research. Like WNV, ZIKV has exhibited an epidemiology characterized by large epidemic outbreaks in some regions but not others. While Colombia and Brazil developed large outbreaks in 2016, we do not currently know if these same countries and regions will be effected to the same degree during subsequent years. Similar to WNV, we may find that development of clinical trials designed to test efficacy of ZIKV vaccines is hampered due to this sporadic epidemiology and relatively rare severe outcomes. Since infection is otherwise mild in adults, then targeted vaccination of women of childbearing age will be a priority. Since only a subgroup of ZIKV infected pregnant women develop fetal infection with associated congenital disease, then efficacy endpoints for ZIKV vaccine deployment will be difficult to develop since some of the more severe and most important complications of ZIKV are not completely known until a pregnancy is completed, and the numbers may be too small to draw conclusions.

As discussed by Zhou et al., ZIKV is also associated with Guillain-Burré syndrome (GBS) following acute infection (Zhou et al. 2017). Since GBS is an immune-mediated phenomenon, it is not clear that a ZIKV vaccine would prevent these potential rare complications. However, prevention of ZIKV infection and spread in epidemic regions by deploying a vaccine may interrupt transmission, decrease infections, and decrease the risk of GBS in regions of ZIKV transmission.

Other potential caveats to ZIKV vaccine development relates to ADE with dengue virus in endemic areas. ZIKV shares several envelope structural features with both WNV and dengue virus and some data suggest that ADE may occur between dengue and ZIKV isolates (Singh et al. 2017). If dengue and ZIKV do exhibit ADE in humans, then this will have several implications. Patients with pre-existing seropositive status for dengue may develop enhanced infection to ZIKV resulting in increased risk of fetal infections in pregnant women. Currently, we know that cell culture assays exhibit evidence of ADE between Dengue and ZIKV. However, ADE has not been demonstrated to occur in an animal model, and we do not currently know if this mechanism is important in human ZIKV pathogenesis. Additionally, the potential for ADE between dengue virus and ZIKV has important implications for ZIKV vaccination programs. There is currently no data regarding the safety of wide-spread vaccination against
ZIKV in dengue endemic regions since theoretically ZIKV vaccination may enhance dengue virus infections. The ongoing phase I ZIKV vaccine trials are critical to understanding some of these questions.

ZIKV surprised many when it was initially associated with sexual transmission. This is another first for an arbovirus. No prior arbovirus has clearly been associated with sexual transmission. While this is likely a minor mechanism of transmission, the risk to pregnant women is real, and the individual impact of sexually transmitted ZIKV to pregnant women is a concern. The CDC has published clear guidelines to protect women who are or may become pregnant from sexualtransmission of ZIKV.

Despite the myriad of challenges associated with the emergence of ZIKV, the global scientific and health-care response has been robust and impressive. In a very short period of time, organizations around the world have sequenced ZIKV isolates, created clones of the virus, developed various vaccine approaches, and developed important epidemiologic real-time monitoring associated with public guidance to prevent spread of infection. Despite the continued development of this epidemic, the modern response to emerging infectious diseases is formidable while still incomplete. Even a single ZIKVinfected child with congenital anomalies is too many. With further study, research, and development, we can improve our responses to future emerging infectious diseases and prevent more of these devastating infections at earlier time points in the epidemic curve. Future development of diagnostic approaches, broadly neutralizing vaccine approaches, broadspectrum antivirals, and neuroprotective treatments may provide the head-start we need against the next emerging virus infection.

\section{References}

Brandler S, Tangy F (2013) Vaccines in development against West Nile virus. Viruses 5:2384-2409

Honein MA, Dawson AL, Petersen EE, Jones AM, Lee EH, Yazdy MM, Ahmad N, Macdonald J, Evert N, Bingham A, Ellington SR, Shapiro-Mendoza CK, Oduyebo T, Fine AD, Brown CM, Sommer JN, Gupta J, Cavicchia P, Slavinski S, White JL, Owen SM, Petersen LR, Boyle C, Meaney-Delman D, Jamieson DJ, Collaboration, U.S.Z.P.R (2017) Birth defects among fetuses and infants of US women with evidence of possible Zika virus infection during pregnancy. JAMA 317:59-68

Singh MV, Weber EA, Singh VB, Stirpe NE, Maggirwar SB (2017) Preventive and therapeutic challenges in combating Zika virus infection: are we getting any closer? J Neurovirol

Zhou K, Wang L, Yu D, Huang, H, Ji H, Mo X (2017) Molecular and cellular insights into Zika virus-related neuropathies. J Neurovirol 